\title{
FABRICATION OF BASIC SCREW EXTRUDER TO MANUFACTURE 3D PRINTERS' FILAMENT
}

\author{
Arvind V. Lal ${ }^{1}$, Monish U.R. ${ }^{2}$, Ahlaad Saraf ${ }^{3}$, Jaya Christiyan K.G. ${ }^{4}$ \\ ${ }^{I}$ M. S. Ramaiah Institute of Technology, Department of Mechanical Engineering, Bangalore, India \\ ${ }^{2,3,4}$ University of Michigan, Department of Automotive Systems Engineering (Department of Interdisciplinary \\ Programs), Dearborn \\ arvind.vlal@gmail.com ${ }^{1,}$ mramakri@umich.edu ${ }^{2}$,ahlaad92@gmail.com ${ }^{3}$
}

\begin{abstract}
The industry of $3 D$ printing in India is fast gathering pace with a large number of $3 D$ printing machines being sold in our country. However there is one major problem that is being faced by almost all the 3D printing customers in the country which is the lack of availability of the $3 D$ printing filament used for $3 D$ printing. Presently the $3 D$ printing filaments are being imported from either US or China. This results in very high overall costs of the filaments due to the high import duties. This reinforces the need to have domestic screw extruders so as to minimize the cost of these filaments. Considering that screw extruders are absolutely necessary in a society where 3D Printing is catching up at a fast pace, it is only a matter of time before domestic screw extruders are made.
\end{abstract}

Keywords : Screw Extruder, Extrusion, 3D Printer Filament

\section{INTRODUCTION}

3D printers use filaments as raw material to build components. Filaments used in these 3D printers are generally made of thermoplastic materials such as Polylactic acid (PLA), Acrylonitrile Butadiene Styrene (ABS), etc. A screw extruder is a device which consists of a screw conveyor with increasing core diameter and variable pitch placed inside a heated chamber. As the screw is rotated the input material is carried along the length of the screw with an increase in pressure due to decrease in the volume. The material is at the same time melted and forced out through the extrusion head. The extrusion head has a nozzle which is shaped according to the requirement. A screw extruder is used to ensure continuous delivery of product.

\subsection{Components of Screw Extruder}

A single screw extruder available in the market for injection molding has the following components:

Screw Conveyor: The screw conveyor is a screw rod with variable core diameter, generally increasing core diameter and with varying pitch or constant pitch. The function of the screw conveyor is to transport the material from the hopper to the extrusion head and also creates s build up in pressure.
It has three sections, feed section, conveying section and metering section.

Drive train: The drive train consists of high torque motor and a gear box. The drive train is used to rotate the screw conveyor at constant speed.

Feeding Unit: The feeding unit is in the form of a hopper which has the capacity to hold enough quantity of raw material for one batch and is designed to provide exact amount of raw material at a constant rate.

Heating System: The heating system uses the principle of inductive heating in order to melt the raw material in the barrel as it is being conveyed along the length of the screw. It can be either clamped onto the barrel or wound on to it.

Casing: The casing is in the form of a steel barrel which encases the screw and supports the bearings which support the screw.

Extrusion Head: The extrusion head is fixed at the end of the screw conveyor. Its function is to compress the molten material and extrude the material in the required form.

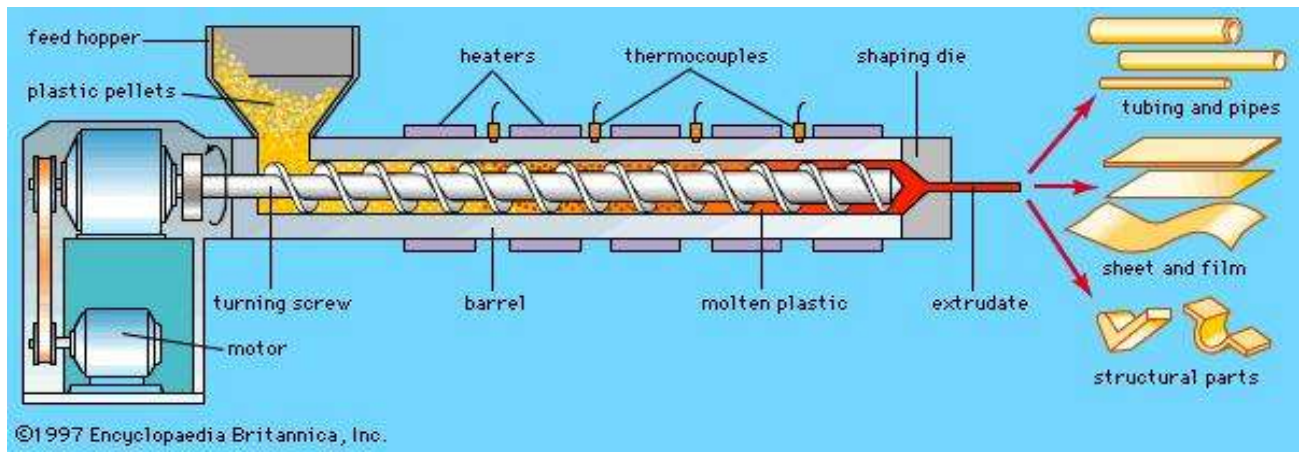

Figure 1: The basic components of a Screw Extruder 


\section{APPROACH AND METHODOLOGY}

\subsection{Advice From Industrial Experts}

Several industrial experts were approached for advice. These were people from the industry who work in the field of injection molding, 3D printing and electrical heating systems. First hand knowledge was gained on the functioning of injection molding systems which use hydraulic pistons or screws to build up pressure. We also obtained invaluable information relating to the various components, heating systems, drive trains that were being used in these machines.

\subsection{Literature Survey}

Existing documents relating to screw extrusion and other related components and parameters were studied. It was mandatory to look at all these papers to obtain all the relevant information that was required for fabrication of the device and designing the screw rod. Some of the referred sources are as follows:

Single Screw Extrusion: Introduction and Troubleshooting: This document provided us with details regarding the screw geometry and the basic dimensions and standards to be followed in order to determine the size of the screw. It gave details concerning the different types of screws available for extrusion and the application of each of these in the industry.

Understanding Plastics Engineering Calculations: This book provided the information regarding the more detailed forms of the screw such as the flight width of the screw and its pitch.

Design of Extrusion Dies: This paper provided us with all the details regarding the theory and the calculations behind determining the dimensions of the extrusion die and the nozzle. It gave a choice between fabricating a complete die and one with a removable nozzle. Considering our requirements of varying filament diameter, we decided to go with determining dimensions for a die with removable nozzle.

Simulation and Design of Extrusion Dies: This was also an important contribution to the theory behind the extrusion die. It provided information regarding the land and the angle range that is followed in choosing the die angle.

Extrusion Principles- Reliance Product Application and Research Centre: it was one of the most important papers that we had to refer during the course of this project. It provided us with most of the theory that formed the basis of this project. It also provided us with details regarding the standards to be followed and the corresponding formulae to be taken while determining screw dimensions.

\section{DETERMINATION OF STANDARDS}

After obtaining information from industrial experts and research papers, the standards based on which the whole system would be fabricated was determined. The standards were chosen based on quality, ease of availability and possibility of mass production. The first parameter chosen for screw design was the standard dimensions of metal pipes in the market which would be used as the casing.

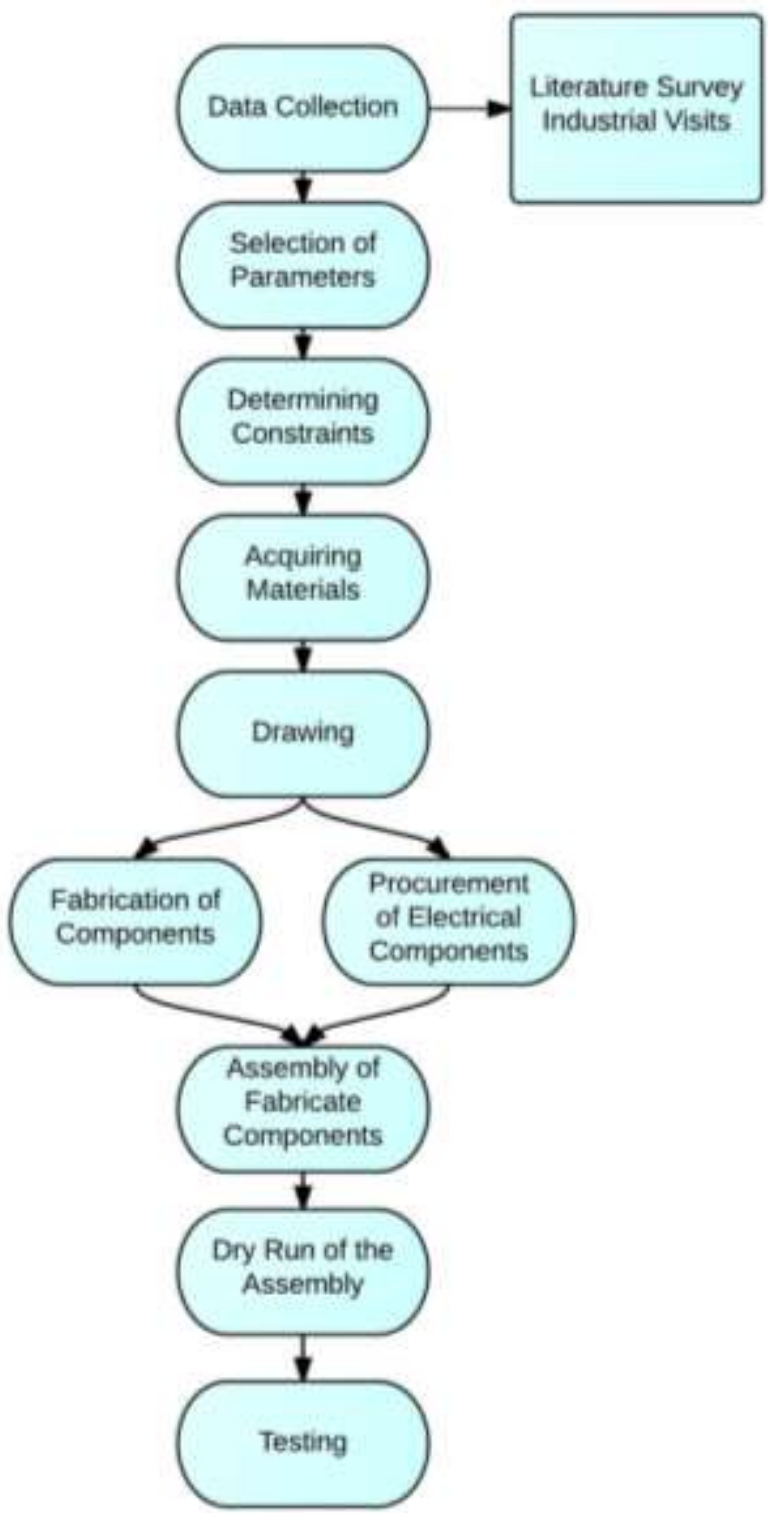

Figure 2: A flowchart depicting the methodology of the project

\subsection{Methodology}

Data Collection: In this stage, we went around collecting information relating to the fabrication of the screw extruder. We took advice from industry experts in the field of extrusion, engineering works and other related machining processes.

Selection of Parameters: After data collection, we had to choose the various parameters that were important in the fabrication process of the screw extruder. These parameters included factors such as casing diameter, bearing selection, heating coil construction, frame construction, motor power determination, and screw dimensions. 
Determining Constraints: This is the stage where we determined all the possible geometric, constructional and supply constraints that we may face during the fabrication process of the screw extruder.

Acquiring Materials: We were able to acquire most of the materials from various shops. While some of the materials chosen were brand new, we have chosen second hand materials or products for other parts such as the frame to support the screw extruder and for the motor.

Drawing: To fabricate the components, we required 2D or $3 \mathrm{D}$ drafts of the components that make up the whole assembly. We have used Autodesk Inventor to model the various components that constitute the screw extruder.

\section{FABRICATION OF COMPONENTS AND}

PROCUREMENT OF ELECTRICAL
COMPONENTS

After the completion of the drawings, we fabricated the components at several engineering workshops with the help of experts. Post fabrication of the mechanical components we procured the electrical heating system. Our design was such that we could assemble the components very easily inhouse. The construction of the frame was simplified to ensure easy mounting of the motor and the screw along with the casing. After assembly, we conducted a dry run of the whole set up so as to ensure that all the components such as the heating system and motor along with screw rod were running smoothly without any complaints. This was the final stage of the project where we tested the screw extruder, to obtain plastic filament through the nozzle.

\subsection{Theory of Extrusion}

Extrusion is essentially a process a material is forced through a die of required dimension and cross section to produce long continuous filaments or other similar products or uniform cross section. Extrusion is widely used in various industries. It is one of the most efficient ways to produce products that are made of thermoplastic input materials. It is also used in producing components that are made of metal.

The screw in the extruder having increasing core diameter rotates and this causes pressure to increase within the chamber which in turn will cause the material to be forced out of the die end with the required cross section. It can be products of varying size or cross section depending upon the specifications of the product. Although, in case of complex cross sections, the expansion of the material post extrusion is also to be taken into account to obtain the exact dimensions.

The extruder barrel or casing is usually divided into multiple heating zones, with a temperature sensor and a controller for maintaining the temperature in each zone. Each controller can supply heat to the zone it has been fixed to using an electric heater which is usually of induction type. The heat to melt the polymer is provided by the heating coils. These coils can be set to the required temperature using the settings on the electric heater.

The feed section conveys the polymer pellets from the feeding hopper to the screw channel, preheats and compacts them, and transports them down the channel towards the die. The compression section however, is the stage where pellets are molten and compacted. In the metering section the polymer melt is homogenized. The pressure is generated and the melt is pumped out of the extruder and through the die. Since the cross section of the die is uniform, the extrusion is uniform and has no breakages or defects of any sort.

There are certain types of screw designs that are made to increase the pressure of the melt flow as it passes through the channel of the screw. The channel depth between the screw and the barrel, $\mathrm{H}$, varies along the length of the screw starting from the feed end to the die end. It becomes smaller from the feed throat to the die. In the compression section, the channel depth between the screw and the barrel decreases gradually to further compact the pellets while at the same time remove any air cavities trapped between them. It also improves heat transfer between the heating coil and the raw material. This melts the polymer raw material and at the same time ensures that the molten polymer does not for an uneven mass by ensuring its proper distribution in the melt. The metering section however has only one major function which is to pressurize the raw material so that it can be forced out of that section into the die. The die contains a nozzle that provides the desired cross section to the polymer filament.

\subsection{Pressure Build Up Inside The Screw Extruder}

During the process of screw extrusion, there is a gradual increase in the pressure of the melt as it passes through the screw channel. As explained previously, this is developed using the screw designs where in the core diameter increases gradually starting from the feed section to the metering end.

The graph below shows the pressure profile along the length of the extruder and the die. At the base of the screw, near the hopper where the raw material is fed initially, the pressure is atmospheric. This pressure increases along the length of the screw towards the die end of the screw and reaches a maximum value just before it enters the die. Once it reaches the die, the pressure reduces back to atmospheric level and reaches this optimum level at the nozzle.

For good extrusion to be achieved, there are 3 basic requirements. One, the polymer should be in a molten state as it reaches the die. Two, the temperature that is maintained by the electric heating system should not be too high as it might cause degradation of the polymer. Three, the pressure that is built up inside the screw casing should be large enough so as to force the polymer out through the small nozzle that is provided. If any of the above conditions are not satisfied, it may lead to improper extrusion or even a case of complete failure of the screw extruder. 


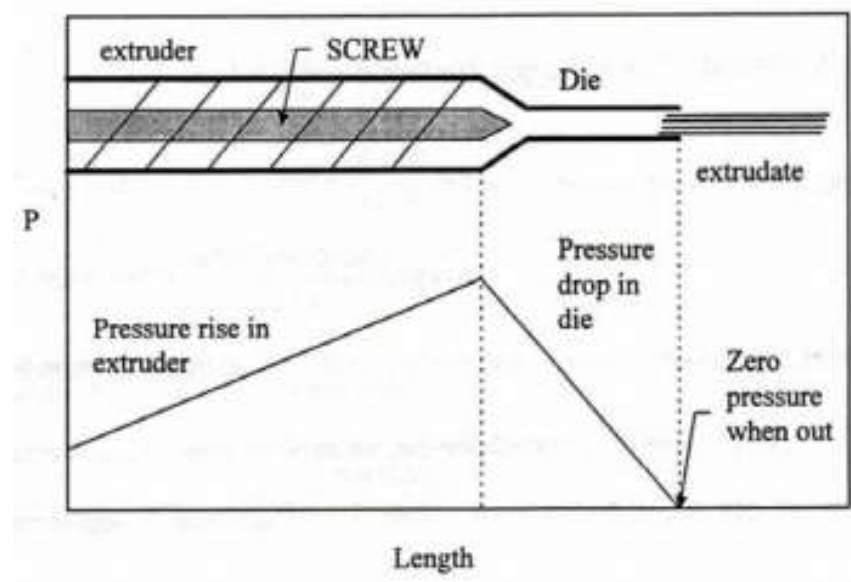

Figure 3: Graph showing the variation of pressure in a screw extruder

\subsection{Design Calculations}

Based on the standard diameters available for the casing, the screw diameter was selected as $40 \mathrm{~mm}$. Another factor considered was that of the thermal expansion of the screw and the casing. The research paper Extrusion PrinciplesReliance Product Application and Research Centre provided us with the formulae relating to the screw geometry and determining the dimensions of the screw. The equations used from this paper were Eqns. marked [7].

Thread diameter, $\mathrm{D}=40 \mathrm{~mm}$

Pitch, $\mathrm{W}=1.0 \times \mathrm{xD} \ldots \ldots \ldots . . .[7]$

$\mathrm{W}=40 \mathrm{~mm}$

Thread Thickness,

$\mathrm{w}=0.1 \mathrm{xD}$.

$\mathrm{W}=4 \mathrm{~mm}$

No. of Flight Starts, $\mathrm{p}=1$

Flight Depth at metering end,

$\mathrm{Hm}=0.1 \mathrm{xD}$.

$\mathrm{Hm}=4 \mathrm{~mm}$

Compression Ratio (Hf/Hm) should around 2-4

2.5 (selected)

$\mathrm{Hf} / \mathrm{Hm}=2.5$

$\mathrm{Hf}=10 \mathrm{~mm}$

Core Diameter at feed end,

$\mathrm{Df}=\mathrm{D}(2 \mathrm{xHf})$

$\mathrm{Df}=20 \mathrm{~mm}$

Core Diameter at metering end,

$\mathrm{Dm}=\mathrm{D}-(2 \mathrm{xHm})$. [7]

\subsection{Expansion Calculations}

\subsubsection{Expansion when Mild Steel was used}

Mild steel was selected for its low thermal expansion coefficient of $8.7 \times 10-6 \mathrm{in} / \mathrm{in}^{\circ} \mathrm{F}$.

$\mathrm{dr}=\alpha \times \mathrm{r} \times \mathrm{dt}$

$\mathrm{r}=0.82 \mathrm{in}$

$\mathrm{dt}=396^{\circ} \mathrm{F}$

$\mathrm{dr}=2.825 \times 10-3 \mathrm{in}=0.0718 \mathrm{~mm}$

This material was abandoned due to rust formation near the thread.

\subsubsection{Expansion when Stainless Steel is in Used}

Stainless Steel was selected in the second attempt due to its moderate thermal expansion coefficient (11.8x10-6 in/in $\left.{ }^{\circ} \mathrm{F}\right)$. After the first attempt where the screw conveyor had gotten stuck inside the casing, the screw rod had been plain turned down to $37.8 \mathrm{~mm}$ diameter to remove the surface damages it had undergone. Due to the reduced diameter of the screw rod, a material of slightly higher thermal expansion coefficient was required to reduce the clearance.

$\mathrm{dr}=\alpha \times \mathrm{r} \times \mathrm{dt}$ [7]

$\mathrm{r}=0.82 \mathrm{in}$

$\mathrm{dt}=396^{\circ} \mathrm{F}$

$\mathrm{dr}=3.832 \times 10-3 \mathrm{in}=0.0973 \mathrm{~mm}$

\subsection{Expansion of Screw}

The screw section was considered to be in the shape of a solid cylinder of diameter $40 \mathrm{~mm}$. The change in dimension due to thermal expansion was considered to be linear along the diameter of this cylinder.

The Eqn 7.10 was obtained from formulae relating to the change in dimensions of components due to increase in temperature. It was obtained from the website www.engineeringtoolbox.com.

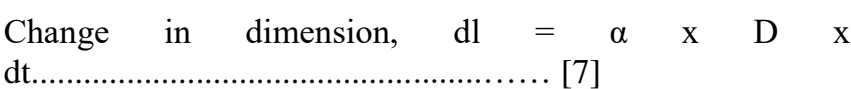

$\alpha=$ co-efficient of thermal expansion $=15 \times 10-6 \mathrm{~m} / \mathrm{mK}$

$\mathrm{D}=0.04 \mathrm{~m}$

$\mathrm{dt}=$ change in temperature $=250-30=220^{\circ} \mathrm{C}$

$\mathrm{dl}=1.32 \times 10-4 \mathrm{~m}$

\section{MATERIALS USED IN FABRICATION}

\section{PROCESS}

EN8 Steel, Stainless Steel, Mild Steel. Die Steel, Brass

\subsection{Device Structure}

\subsubsection{Screw Conveyor}

The screw rod of constant pitch $40 \mathrm{~mm}$ and varying core diameter from 20 to $32 \mathrm{~mm}$ is made from EN-8(Euro Norm$8)$ medium carbon steel. The outer diameter of the screw thread is made to $37.8 \mathrm{~mm}$. The thread width is $4 \mathrm{~mm}$ and the helix angle is $18^{\circ}$. The length of the screw conveyor is 500 $\mathrm{mm}$ and is divided into three sections, that is the feed section, conveyor and melting section and finally, the metering section. The material is fed into the casing on the screw blade in the feed section of the screw rod. The material is pushed along the length of the screw and melts due to the heat supplied. The design of the screw rod causes a build up of pressure as the channel width decreases. The high pressure molten material is then pushed towards the extrusion head. The screw has an inbuilt washer to ensure back flow of molten plastic material does not affect the functioning of bearings. To ensure easy maintenance, the bearing is made to be easily accessible. The bearing can be 
easily removed by opening the nut screwed into the screw. The drive train in our device consists of a three phase induction motor with an inbuilt gear box. The motor runs at a speed of $1350 \mathrm{rpm}$. The internal gear box has a ratio of 55:1 and runs the drive shaft at $25 \mathrm{rpm}$. The drive shaft is used to rotate the screw conveyor. The drive train was bought as a set from the market. In the device the feeding unit consists of a hopper. The hopper is made of Galvanized Iron (GI) sheets. In our initial testing, we have found that the hopper is getting heated if it remains in contact with the casing. This results in the material melting partially and blocking the entry point or preventing smooth flow of pellets. To avoid this, the industry experts have advised us to provide a cooling system at the hopper, however, this is time consuming and expensive. Based on industrial advice the temperature selected is $220^{\circ} \mathrm{C}$. A control box is used to maintain the temperature using an auto cut off system. The heat is supplied from a single phase $2 \mathrm{KW}$ source to 4 heating coils of $100 \mathrm{~mm}$ length and $0.5 \mathrm{KW}$ power each. Each heating coil is in the form of a clamp to facilitate easy assembly and removal and also to allow a tolerance in the diameter. The heating coil is made up galvanized iron sheets which sandwich a mica sheet. In order to provide a constant heat supply a control box consisting of fuse, 15 amp circuit breaker, 2 indicator lamps ( 1 red and 1 green), a temperature monitor to view and set the temperature and a contactor coil is also provided. The Casing or the barrel encompasses the screw conveyor and holds the heating coils on the outside. The casing should have sufficient strength and stiffness to withstand the internal pressure generated due to the compression of molten plastic along the length of the screw conveyor. The casing should also be able to support the load of the screw conveyor which is resting on the casing at temperatures of up to $260^{\circ} \mathrm{C}$. Considering the above constraints and the standard sizes of pipes available in the market we chose a stainless steel pipe of internal diameter $41 \mathrm{~mm}$ and external diameter $51 \mathrm{~mm}$. The material used for the casing stainless steel. The casing dimensions were selected on the basis of ASTM. The thickness as compared to that use in similar industries is much lower so that the heat transferred to the insides involves lower losses. To facilitate this lower thickness of the casing the extruder is aligned vertically instead of the typical horizontal position. The extrusion head is made of die steel and the nozzle is made of brass. The length of the extrusion head is $60 \mathrm{~mm}$. The internal surface of the extrusion head is in the form of a cone of $45^{\circ}$ taper angle and is used to direct the material towards the nozzle. The internal face of the extrusion head is threaded so as to fix it to the casing while at the same time enabling it to be a replaceable piece. The nozzle is screwed into the center of the extrusion head. The nozzle is also designed to be replaceable so that different diameters of filament based on the required standards can be produced without changing the entire set up of the assembly.

\subsection{Fabrication process}

\subsubsection{Fabrication of Screw Rod}

The Screw Rod is fabricated using a single rod of EN8 (Euro Standard 8) medium carbon Steel (Unalloyed Steel which is produced by cold drawing). The processes carried out on the rod to produce the screw rod include Facing, Plain Turning, Threading, Grinding

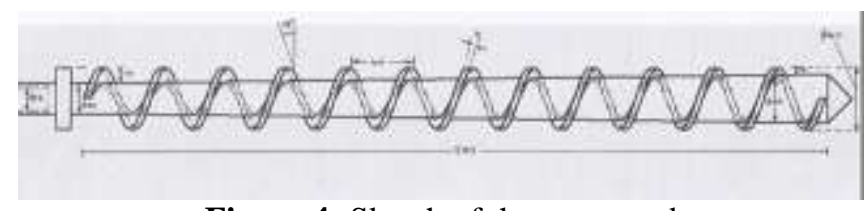

Figure 4: Sketch of the screw rod

\subsubsection{Fabrication of Extrusion Head}

A cylindrical block of die steel was fixed on to a lathe with a 4 jaw chuck. The stock of die steel was aligned with the center of the lathe axis so as to ensure concentricity of the stock material before and during the machining operation. The die steel block was then faced on both sides after which a hole was made on one of the sides for seating the tail stock.

Plain turning, Boring operation, Internal taper turning, Threading

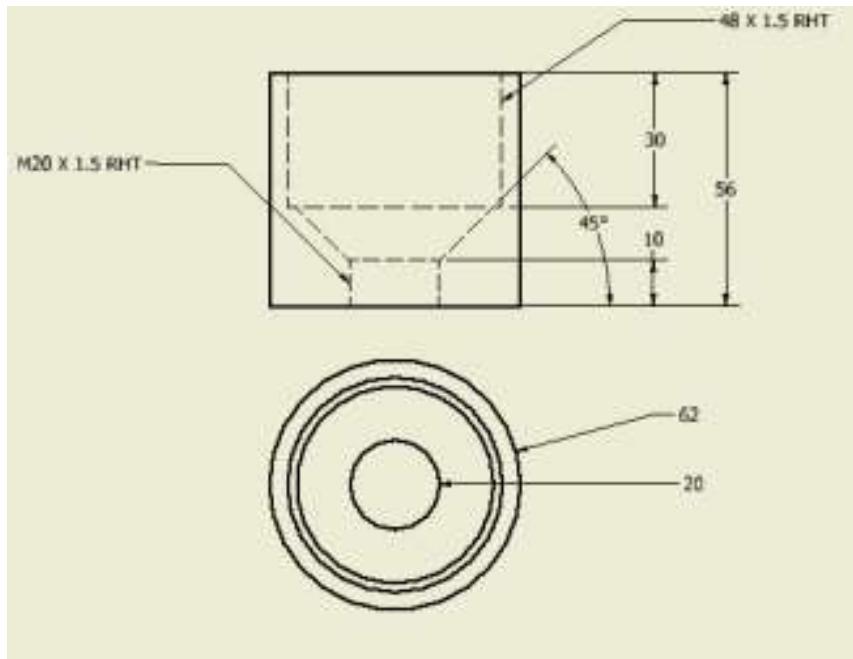

Figure 5: Sketch of Extrusion head

\subsubsection{Fabrication of the Nozzle}

The nozzle is screwed on to the extrusion head and is made out of brass. The brass stock piece which was initially obtained at a standard diameter of $25.4 \mathrm{~mm}$ and then subjected to various machining operations.

Facing, Plain turning, Step turning, Drilling, Threading 


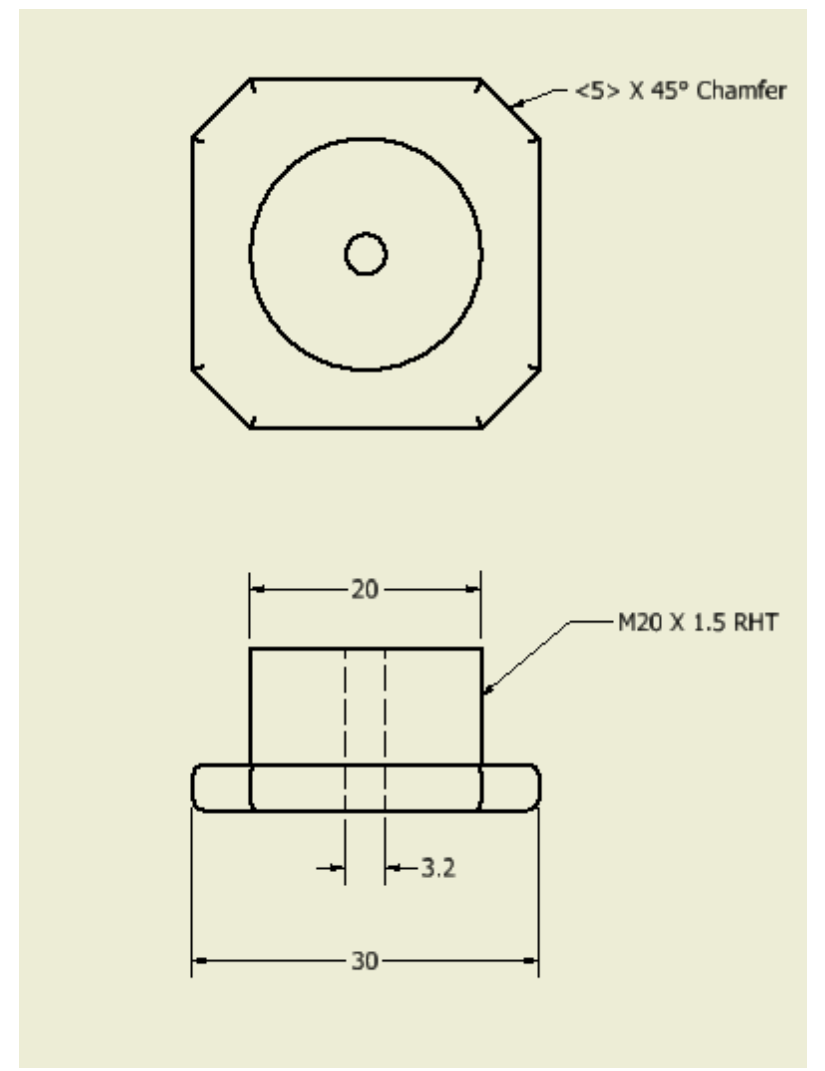

Figure 6: Sketch of the nozzle

\subsubsection{Fabrication of the bearing support}

The bearing support was made of die steel and is mainly used to keep the thrust bearings in their place and also to provide support to the screw while it rotates during operation of the screw extruder.

Facing, Boring, Internal Turning, Internal threading

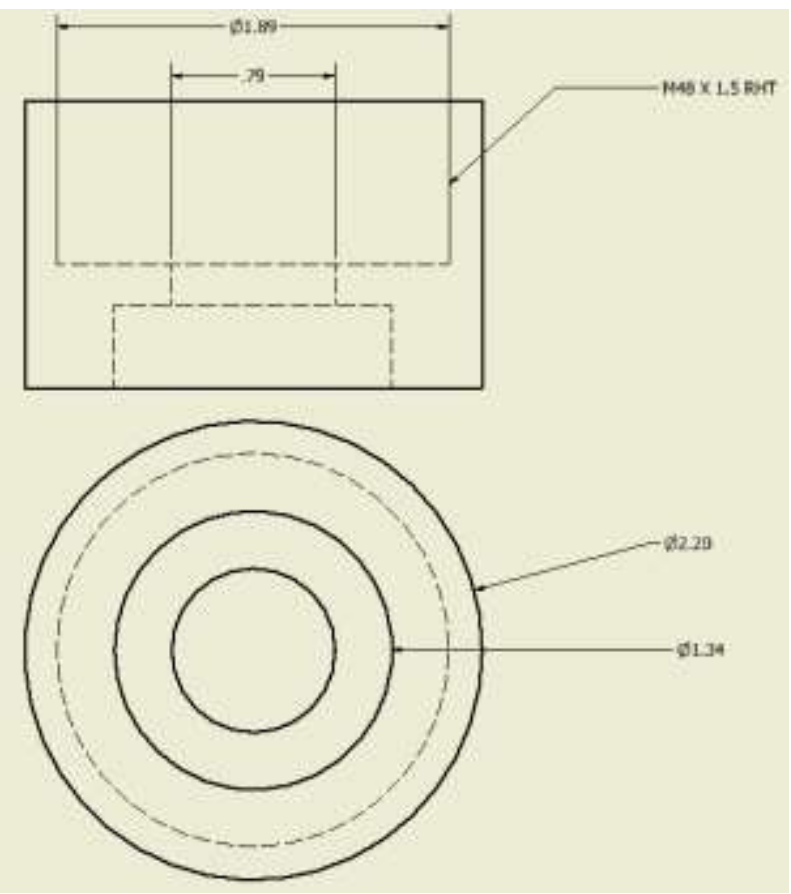

Figure 7: Bearing support sketch

\subsubsection{Fabrication of Screw rod stud}

The stud was fabricated to ensure that the screw does not fall through the casing during the operation. The stud is screwed on to the end of the screw during rotation so that it holds the screw near the cap without allowing it to loosen.

Plain turning, Step turning, Threading
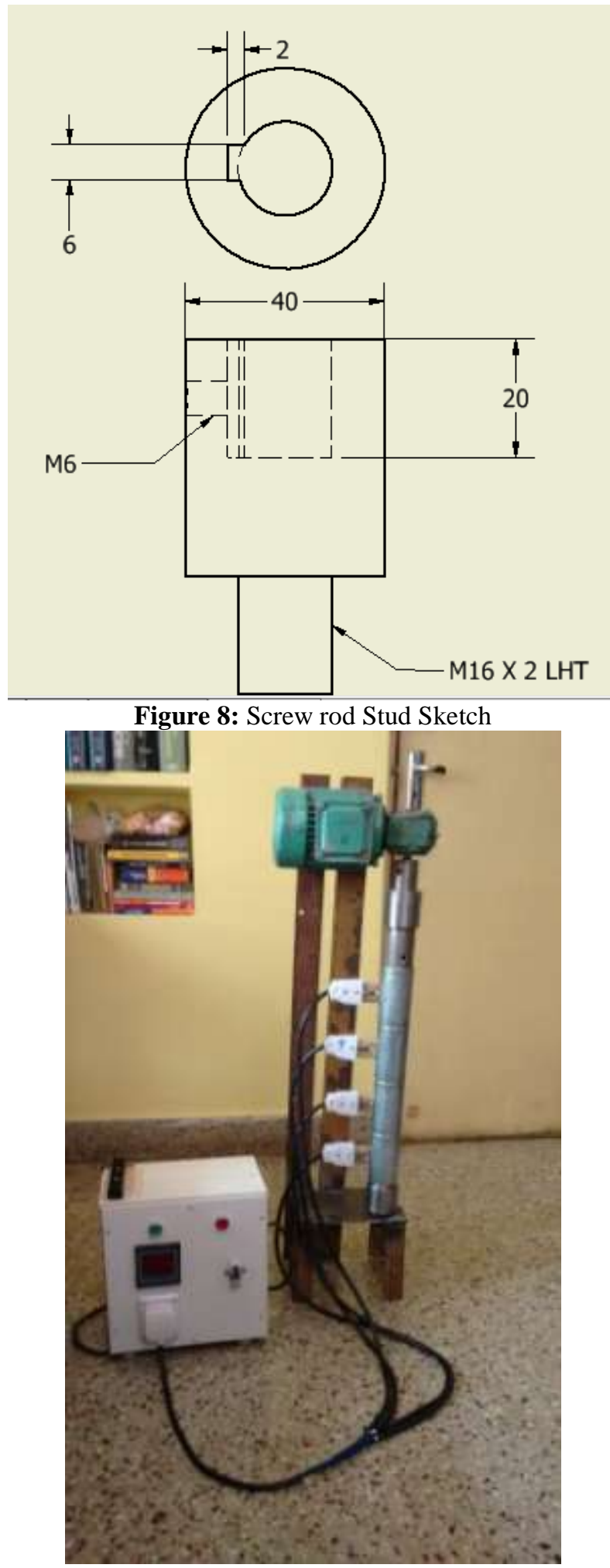

Figure 9: A photograph of the completed Screw extruder 


\subsection{Testing and Analysis of the Device}

\subsubsection{Preliminary test}

In the first test that was carried out, we ensured the stability of the entire assembly on the frame we had fabricated. The power train and the screw conveyor along with the die and the casing were assembled on to the frame, after which we connected the motor to a 3 phase supply and switched it ON. This was done in order to observe any structural defects in the frame and to check the rotation of the screw rod in the assembly without any obstruction.

\subsubsection{Observations and analysis}

It was observed that the frame was rigid and exhibited no vibration or weaknesses at any part of the frame. Hence we concluded that the frame had the structural integrity to withstand the various loads that were generated during the working of the device.

Coming to the second objective of our test, we observed that the screw scraped the inner surface of the casing at one point. Upon disassembling the unit, we found that there was a mismatch in the concentricity of the casing and the screw rod. Also, due to an anomaly during the process of chrome plating, three threads on the top section of the screw had a thicker coating than the rest of the threads of the screw.

\subsubsection{Modification}

In order to rectify this problem, we used a file to remove the extra material and reduced the thickness of the layers of the defective threads to the required dimensions. We filed the chrome plating on the screw to the extent where the screw rod could rotate freely inside the casing without scraping its inner sides.

\subsubsection{Preliminary Heating coil test}

Preliminary heating test was carried out to check whether the electrical systems that were procured for the purpose heating the casing of the screw conveyor assembly and the motor system for rotating the screw during operation was working without any issues. The first stage was to check the working of the motor that we had bought. The motor we procured was a second hand motor that was about 10 years old so we had to ensure that the motor was running smoothly and was providing the required torque at the specified rpm. The second stage of our testing included checking the heating system and its functioning.

\subsubsection{Observations and analysis}

The motor was wired to a 3 phase supply and power was switched on. It was observed that the motor was rotating at a uniform rate and was rotating the screw inside the casing. It was also observed that there were no strong vibrations generated during rotation of motor. Also, there was no heat generated during the operation of the motor over a period of time.
The Heating system was plugged into single phase 15 Amps socket and the heating coils were wired and plugged into the control box. After this, four thermocouples were connected to the control box at one end and the casing on the other. The power supply was switched on and then, the switch on the control box was switched on. We then set a temperature of $150^{\circ} \mathrm{C}$ on the monitor and observed the rise of temperature and functioning of the auto cut off feature. It was observed that auto cut off feature, switched off the power supply when the set temperature was reached, however, the temperature continued to rise till about $185^{\circ} \mathrm{C}$ and then reduced to $150^{\circ} \mathrm{C}$. After this the temperature stabilized at $150^{\circ} \mathrm{C}$. Hence, we concluded that the electrical systems were functioning correctly.

\subsubsection{Dry Run of Device}

The entire device was set up and the necessary connections were made. The power was switched on and initially the rotation of screw rod was observed. After this, the temperature was set at $22^{\circ} \mathrm{C}$ and the functioning of device was observed.

It was observed that the motor rotated the screw rod freely up to about $100^{\circ} \mathrm{C}$. At this temperature, the screw rod started to rub with the casing and eventually got jammed inside the casing. At this point the motor rotated the casing along with the screw rod. Supply was immediately switched off to ensure that there was no damage to the heating system. Upon disconnecting the heating system, we tried to remove the screw rod from the casing. This could not be done, even after cooling it down as the screw rod had completely jammed inside the casing. We had to cut the casing out using power tools to remove screw rod from it. We had to purchase another steel pipe for our casing. This time we chose a Stainless steel Pipe of $41 \mathrm{~mm}$ internal diameter and $51 \mathrm{~mm}$ outer diameter. We chose a SS pipe as it had moderate thermal expansion coefficient, does not rust and we were able to procure a pipe of required dimensions. After this turning, threading, drilling and honing operations were carried out as explained previously.

\subsubsection{Final Test}

This is final test of the device we have carried out as on date the report was prepared. The aim of this test was to observe the functioning of the device with the addition of thermoplastic material. For the test we procured $1.5 \mathrm{~kg}$ of PLA in the form of virgin pellets. Upon switching on the device, we heated the melt chamber to $200^{\circ} \mathrm{C}$ and ensured that the chamber was maintained at this temperature. After this, we used a metal hopper to pour the thermoplastic pellets in to the chamber through the opening. It was observed that though initially there was smooth flow of pellets, but after some time, the hopper got heated up and the pellets started to soften in the hopper. This clogged the hopper. To avoid this, the metal hopper was removed and we used a cardboard as a funnel to pour material in to the chamber. This ensured smooth flow of the pellets. The screw rotated in the casing uniformly and the temperature was also observed to be at the same point. The same 
conditions were maintained and material was fed continuously. There was no extrusion of filament and as a result we stopped the device and removed the nozzle after the device was cooled to check for the reasons why there was no extrusion of filament. We found that the PLA had melted and reached the top of the nozzle, where it solidified. We had concluded that the following reasons may have factored:

a) Insufficient Pressure

b) Lower Temperature at nozzle than required

c) Insufficient rate of addition of feed material

Upon restarting the machine, we first attached a thermocouple at the nozzle region. It was observed that the nozzle region was on an average $30^{\circ} \mathrm{C}$ lower than the melt chamber. Upon removing this solidified material from the extrusion head, we found that the amount of feed was sufficient. We decided to remove a heating coil we had attached on the casing that was near the hopper and placed it around the extrusion head.

\section{FINAL RESULT}

The final modification carried out on the set up helped solve the problem of the hopper getting heated as well as the lower temperature at nozzle region. This resulted in the working of the screw extruder and provided filaments that were close to the required diameter.

\subsection{Estimated Cost of Project}

The material costs for the fabrication of this type of extruder came up to roughly Rs. 5000 while the machining costs came to Rs.7250. the pre fabricated components cost Rs. 10575 .

Grand total $=$ Material cost + Machining cost + Prefabricated components cost

Grand Total $=5000+7250+10575=$ Rs $22825 /-$

\section{CONCLUSIONS}

The aim of this project was to fabricate a screw extrusion device that could manufacture 3D printer filament within the time constraints imposed by our course. Over the three months we have tried to achieve this goal. We had several obstacles along the way due to defects in materials used, insufficient heating or an assortment of other problems which lead to solidification of material at nozzle.

\section{REFERENCES}

[1] Friction Properties of Thermoplastics in Injection Moulding Ferreira, E.C., Neves, N.M., Muschalle, R. and Pouzada, A.S.

[2] Single-Screw Extrusion: Introduction and Troubleshooting-Cambell and Spalding

[3] Shigley's Mechanical Engineering Design Tutorial 3-16: Press and Shrink Fits

[4] Modelling of Melt Pressure Development in Polymer Extrusion: Effects of Process Settings and Screw Geometry
-Chamil Abeykoon, Kang Li, Peter J. Martin and Adrian L. Kelly

[5] Guidelines for the Extrusion of Sarlink Screw design basics Andrew W. Christie

[6] Simulation and Design of Extrusion Dies

-Kirubel Bogale

[7] Extrusion Principles Reliance Product and Application Center, Mumbai

[8] Understanding plastics and engineering calculations Natti S.Rao and Nick R. Schott

[9] Design of extrusion dies Milivije M. Kostic and Louis G. Reifschneider

[10] Extrusion moulding methods A. Lopez

[11] The design of extrusion screws: An optimization approach A. Gazpar-Cunhe and J.A. Covas

[12] Multi-staged vented extrusion screw with dual channel depth pumping section Patent Number: US 20020136084 A1

\section{ACKNOWLEDGMENT}

The authors greatly appreciate and thank Asst. Prof Rajendra P. and Asst. Prof. Jaya Christiyan K.G., for providing us with valuable guidance and support. 